Proceeding Paper

\title{
Virtual Models for Crystallography Teaching in Mineralogy: Some Suggestions ${ }^{+}$
}

\author{
Carlos Alves ${ }^{1, *}$, Carlos Figueiredo ${ }^{2}$ and Jorge Sanjurjo-Sánchez ${ }^{3}$ \\ 1 LandS/Lab2PT-Landscapes, Heritage and Territory laboratory (FCT-UIDB/04509/2020) and Earth Sciences \\ Department/School of Sciences, University of Minho, 4710-057 Braga, Portugal \\ 2 CERENA-Centro de Recursos Naturais e Ambiente, FCT-UIDB/04028/2020, DEcivil, Instituto Superior \\ Técnico, University of Lisbon, 1049-001 Lisbon, Portugal; carlos.m.figueiredo@ist.utl.pt \\ 3 Instituto Universitario de Xeoloxía, Universidad de la Coruña, ESCI, Campus de Elviña, \\ 15071 A Coruña, Spain; jorge.sanjurjo.sanchez@udc.es \\ * Correspondence: casaix@dct.uminho.pt \\ + Presented at the 3rd International Electronic Conference on Geosciences, 7-13 December 2020; \\ Available online: https://iecg2020.sciforum.net/.
}

Citation: Alves, C.; Figueiredo, C.; Sanjurjo-Sánchez, J. Virtual Models for Crystallography Teaching in Mineralogy: Some Suggestions. Environ. Sci. Proc. 2021, 5, 10. https://doi.org/10.3390/IECG202008738

Academic Editor: Jesus Martinez Frias

Published: 1 December 2020

Publisher's Note: MDPI stays neutral with regard to jurisdictional claims in published maps and institutional affiliations.

\section{Copyright: (c) 2020 by the authors.} Licensee MDPI, Basel, Switzerland. This article is an open access article distributed under the terms and conditions of the Creative Commons Attribution (CC BY) license (http://creativecommons.org/licenses /by/4.0/).

\begin{abstract}
Crystallography concepts are usually among the most demanding subjects for Mineralogy students. Traditional onsite teaching of Mineralogy starts with physical models of crystal polyhedra and frequently also includes the observation of models of crystal structures. These teaching strategies could be difficult to implement under pandemic situations like the present one. But they also have other disadvantages under the usual access conditions as their use by the students is restricted by the number of students in relation to the number of models and by the availability of the models and teaching staff. Additionally, onsite teaching can pose challenges to both students and teachers with temporal or permanent disabilities. We consider here some possibilities of teaching with virtual models of crystal polyhedra, twinning, and crystal structures, based on some of the available freeware options and considering the main concepts taught in the usual Mineralogy syllabus.
\end{abstract}

Keywords: polyhedra models; crystal structures models; distance teaching; COVID-19

\section{Introduction}

The current pandemic situation has caused disruptions in many activities around the world, including teaching. The promotion of physical distancing procedures becomes especially strenuous for subjects mostly based on laboratory work since they have higher requirements in terms of spaces and materials.

Crystallography concepts are usually among the more demanding subjects for Mineralogy students. Traditional onsite teaching of Mineralogy starts with physical models of crystal polyhedra and twinning and frequently also includes the observation of models of crystal structures. These teaching strategies could be difficult to implement under pandemic situations like the present one. But they also have other disadvantages under the usual access conditions as their use by the students is restricted by the number of students in relation to the number of models and by the availability of the models and teaching staff. Additionally, onsite teaching can pose challenges to both students and teachers with temporal or permanent disabilities.

We consider in the present work some possibilities of using virtual models for teaching some crystallographic concepts usually deemed necessary for the study of mineral characteristics.

There have been several reviews of software dedicated to Mineralogy, and a recent one dedicated to crystal shapes and structures is presented in Rakovan [1]. It is not our intention to present a software analysis or review, nor are we implying that the software 
programs mentioned in the following examples are the only option or even the best ones. The ones presented here are the ones that we know and that we have used, and all software referred to here is freeware; the interested reader can easily download the software (links for download are available in the references).

\section{Materials and Methods}

All the presented work was prepared in a Windows-based computer, and we present next an alphabetically ordered list with the software used and their reference for easier consultation by the reader:

+ ) KrystalShaper 1.4.0 [2];

+) PowderCell 2.4 [3];

+ ) VESTA 3.5.2 [4];

+ ) WinXMorph $[5,6]$.

For any of the software examples considered in our analysis, the students can simply read files that are available in public repositories or that were prepared by the instructors, or they can build the models from basic crystallographic principles, which will be indicated in the next section where the examples are discussed.

We must also refer to an important source of information for most of the work prepared in the context of this paper: The AMCSD (American Mineralogist Crystal Structure Database [7]).

There are also options allowing to see crystal models online such as, for example, http://webmineral.com/ and https://chemistry.beloit.edu/edetc/pmks/index.html (accessed on 21 November 2020), but our discussion will be focused on examples where the user can create, keep and modify the files for the models.

We will analyze the use of these software examples in the teaching of crystallography concepts usually taught in Mineralogy undergraduate courses, considering namely models of internal crystal structures and models of crystal forms (including twinning).

\section{Examples and Discussion}

\subsection{Models of Crystal Structures}

The AMCSD [7] has a series of files with information on structures of minerals that can be accessed online. These files can be downloaded and open in software such as VESTA [4] that immediately prepares virtual 3D models of the structures. This software allows diverse types of representation such as ball-and-stick, sphere packing, and coordination geometries (and it is very easy to indicate to the students a few commands for the visualization options).

The first author has verified that students can prepare suitable images from models in a matter of a few minutes, even when teaching through a chat. An example is presented in Figure 1a, showing an image of a model for the structure of sillimanite based on a file available in the AMCSD [8]. In this image, it is used a representation by coordination polyhedra showing, for example, isolated silicate tetrahedra and that aluminum has two different coordination geometries: octahedral (Al1) and tetrahedral (Al2). While the figure is a 2D object, VESTA produces 3D virtual objects that can be manipulated. While the software allows the instant creation of the model from the reading of the downloaded file, the user still has a lot of potential options that can be used to discuss different issues (labels and colors of atoms, presenting just some of the atoms or some of the coordination polyhedra, etc.).

It is also possible, and perhaps more interesting, for teaching the concepts associated with mineral structures to create models from scratch. We present in Figure $1 b, c$ images from a model of the structure of graphite [9] prepared with PowderCell [3], which also produces 3D models. This example will be useful to relate the perfect and easy cleavage of graphite to its structural characteristics. The experience of the first author shows that first-year students can build these relatively simple models quickly (in a matter of some 
minutes). Afterward, they can build models of more complex structures, which is, essentially, a matter of time given that it is necessary to introduce the positions of a bigger number of atoms.

It is also possible to present portions of a cell unit or with several cell units, as well as to show just some of the chemical elements.

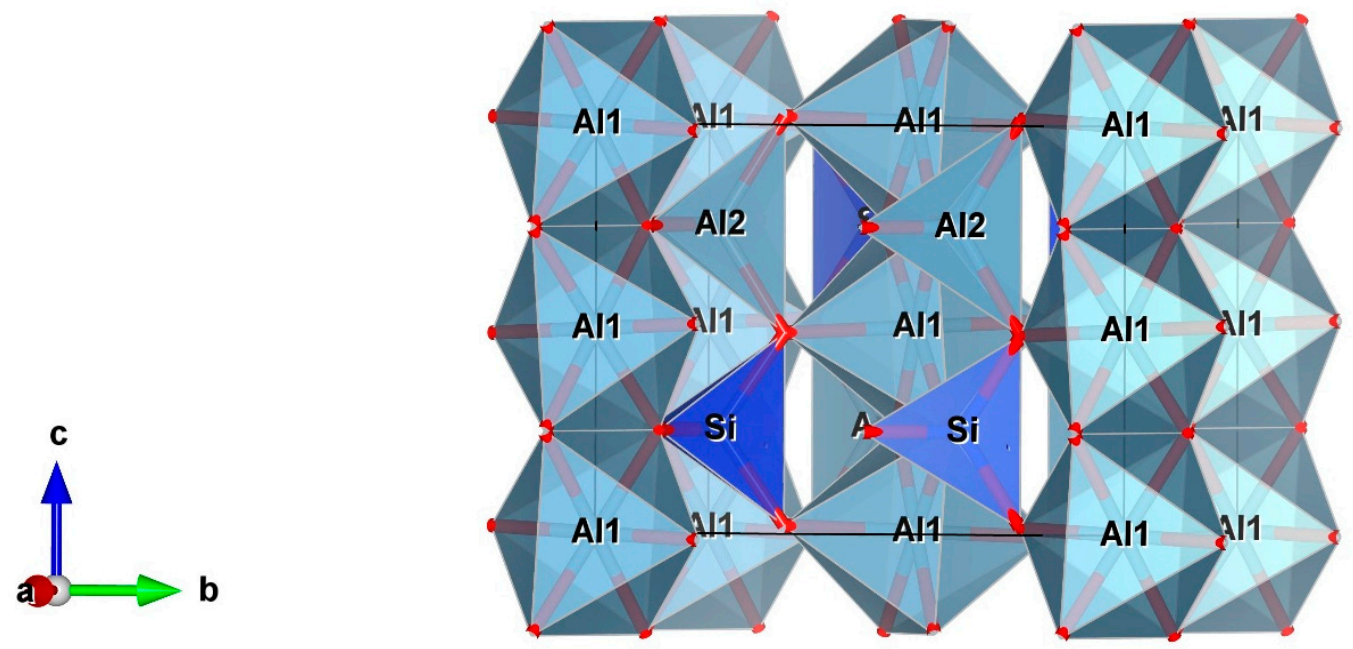

(a)

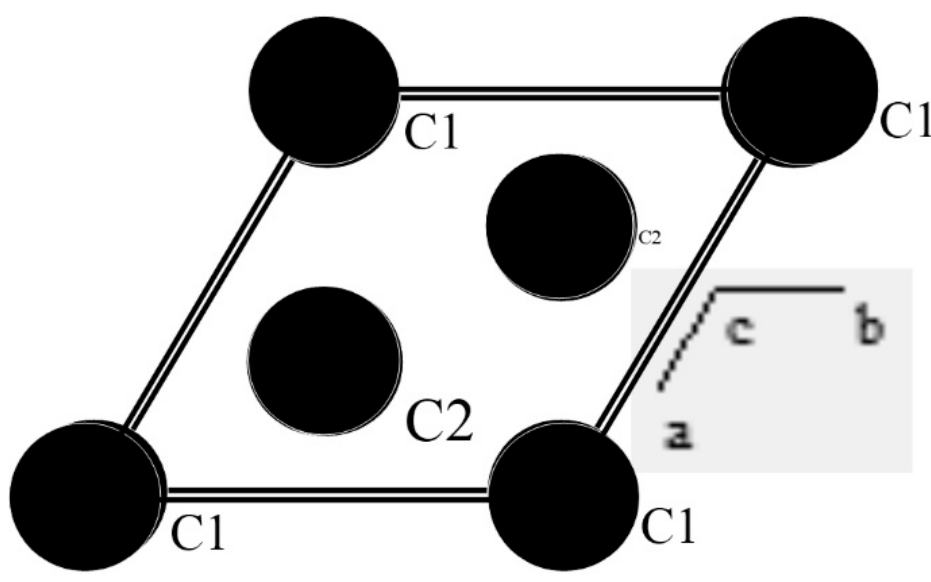

(b)
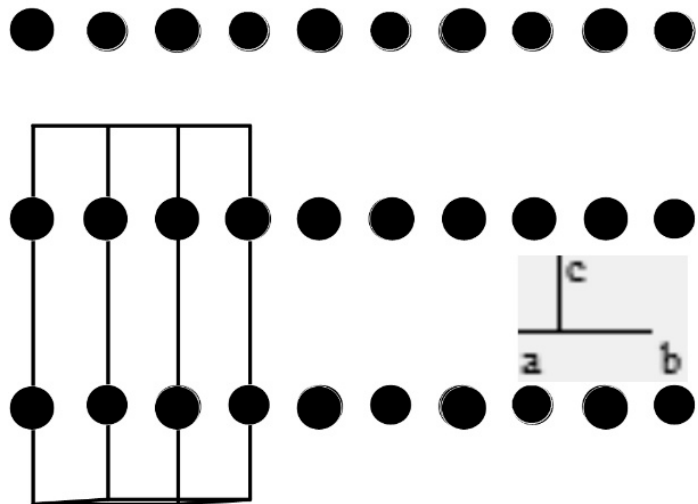

(c)

Figure 1. Images of models of crystal structures: (a) structure of sillimanite [8] obtained with VESTA [4] showing coordination polyhedra of $\mathrm{Si}$ (tetrahedral) and $\mathrm{Al}$ (octahedral), as well as the chains of $\mathrm{Al}$ coordination polyhedra and the isolated silicate polyhedra (typical of nesosilicates); (b,c) images of graphite structure [9] prepared with PowderCell [3] observed in a normal view to (001), showing the hexagonal cell and the two structural positions of carbon atoms (b) and with a view parallel to (001) showing the layered distribution of atoms that explain the easy pinacoid (basal) cleavage of this mineral.

\subsection{Models of External Morphologies (Crystal Forms and Twinning)}

The study of crystal shapes in models where the faces have ideal proportions (frequently in materials such as wood) is a classic foundation stone of mineralogical teaching. This is useful both to better introduce students to certain concepts (reducing the variables that are studied in the beginning) and to promote a visual familiarization of the student with crystal shapes that in real mineral samples can occur distorted or truncated.

There are freeware software examples that can create virtual models of crystal forms, such as KrystalShaper [2], which only needs information on crystal class, the Miller symbol of the crystal form, and if necessary, the characteristics of the unit cell. The created 
model can be rotated freely as if it was a physical one. This works especially well for cubic crystals since all forms are closed (for the other crystal systems, the models are created only when the crystal form or forms define a closed polyhedron).

There are options allowing to show the Miller symbols of the different faces and the normal to the faces (which will be helpful as a first step in the teaching of stereographic plotting).

Another useful feature of this software is the possibility to show students how the same Miller symbol could produce different crystal forms in different crystal classes, such as $\{111\}$, which, for example, produces an octahedron in class 432 , a tetrahedron in class 23 , a hexagonal bipyramid in $6 / \mathrm{m}$, etc.

It is also possible in KrystalShaper to attribute different importance to the crystal forms, which could be used for a (limited) illustration of cleavage effects. In Figure 2, we present an example that attempts to expand the use of KrystalShaper to show the effects of cleavage considering a cubic crystal of fluorite with octahedral cleavage. For that, we consider the example of fluorite and the combination of cube and octahedron, used here to represent the $\{111\}$ cleavage, with different proportions, from a cube with truncated corners (due to cleavage) to the final cleavage octahedron.

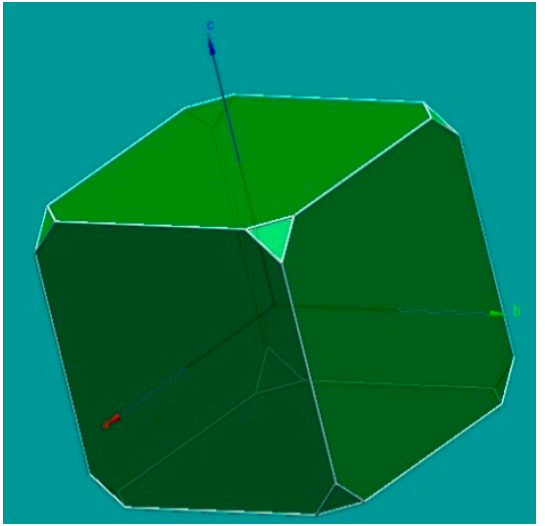

(a)

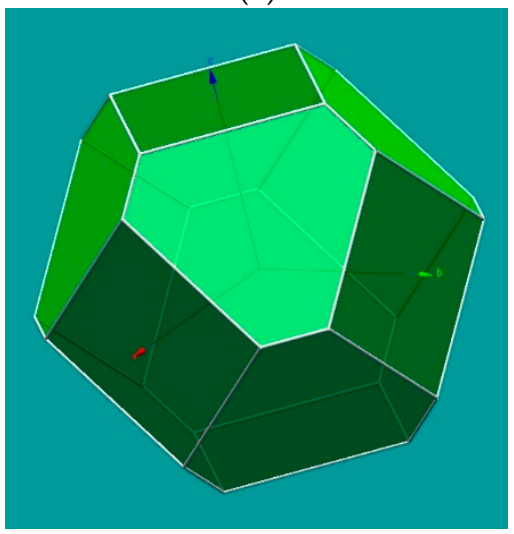

(c)

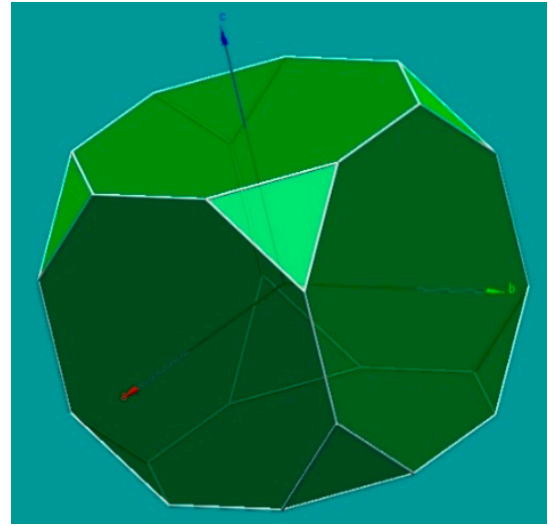

(b)

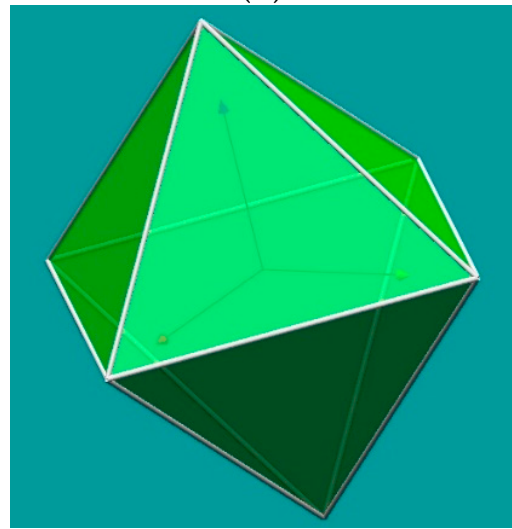

(d)

Figure 2. Images of a model of $\{100\}$ and $\{111\}$ in fluorite prepared with KrystalShaper [2], with different situations of the relative importance of these crystal forms, aiming to simulate the effect of the octahedral cleavage in fluorite, from little importance in (a), where octahedral cleavage cuts the corners of a cubic crystal, with $(\mathbf{b}, \mathbf{c})$ representing further effects of cleavage and, finally, (d) corresponding to the cleavage polyhedron obtained for fluorite.

This software allows the user to attribute different colors to different crystal forms, something useful to show the concept of complementary forms and how they can create examples of pseudosymmetry, such as, for example, complementary rhombohedra of quartz creating a pseudohexagonal bipyramid. Another point concerns the relation of the 
considered crystal forms to the geometric characteristics of the unit cell and the Miller symbols, which can be useful for presenting other pseudosymmetry examples such as the pseudo-octahedral scheelite and pseudo-tetrahedral chalcopyrite.

KrystalShaper has the additional advantage of showing the stereographic projection of the crystal forms (with the option of presenting the Miller symbol of each pole). It is possible to show the change in the position of the stereographic poles with the rotation of the model (which, as mentioned above, could show the lines that are normal to the faces). These features can be used in the discussion of the symmetry elements of the model.

There are also software options for making models of crystal twinning. WinXmorph $[5,6]$ is an easily accessible freeware that can be used for that end (this software can also prepare models of crystal forms from scratch and reading from available files); Figure 3 presents a model of fluorite twinning prepared with this software.

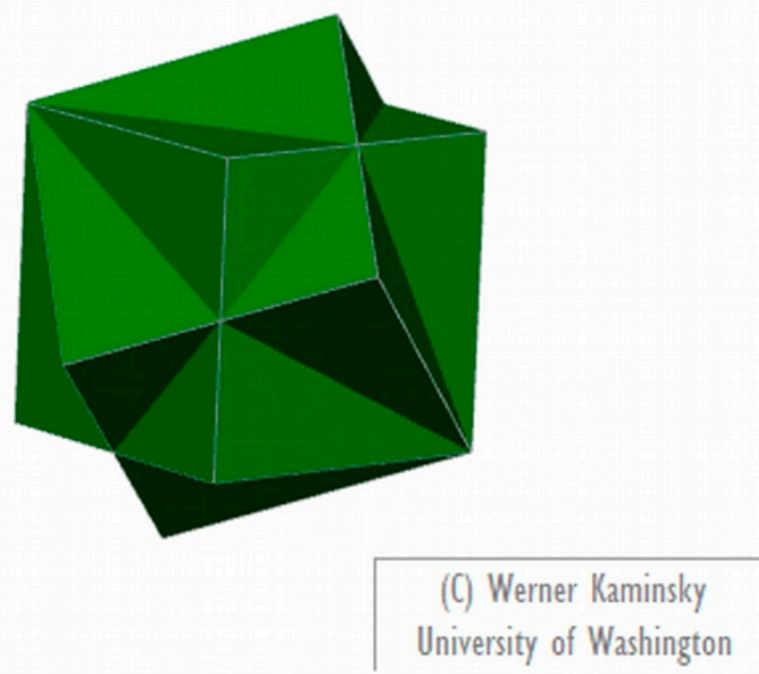

Figure 3. Image of a model of fluorite twinning prepared with WinXMorph $[5,6]$.

\section{Conclusions}

While the software examples considered in this work were few, they all produce 3D objects that can be freely rotated and used as models and that show high potential for teaching the main crystallographic concepts usually required in traditional Mineralogy lectures, both in terms of structures and crystal morphology (crystal forms and twinning).

Author Contributions: All authors contributed to the present paper. All authors have read and agreed to the published version of the manuscript.

Institutional Review Board Statement: Not applicable.

Informed Consent Statement: Not applicable.

Data Availability Statement: Data sharing not applicable.

Acknowledgments: We would like to thank first and foremost all the authors of the freeware used for the preparation of this paper (more details in Materials and Methods and in the references). The Lab2PT/Landscapes, Heritage and Territory laboratory -UIDB/04509/2020 is supported by the Portuguese FCT - "Fundação para a Ciência e a Tecnologia". The authors also gratefully acknowledge the support of the CERENA (funded by a strategic project of the FCT-UIDB/04028/2020) and the LAMPIST of the DECivil, Instituto Superior Técnico, University of Lisbon, Portugal. The University Institute of Geology of the University of A Coruña (Spain) receives support from the Xunta de Galicia from the program "Consolidación y estructuración de unidades de investigación competitivas: Grupos de potencial de crecimiento" (ED431B 2018/47) and Redes de investigación (R2017/008). The first author thanks his first-year students of the curricular unit "Mineralogia Complementar" of the Geology BSc at the University of Minho in the 2019/2020 academic year for their valuable input, 
especially Enzo Cruz and Lourenço Fernandes for insights regarding VESTA and KrystalShaper, respectively.

Conflicts of Interest: The authors declare no conflict of interest. The founding sponsors had no role in the design of the study; in the collection, analyses, or interpretation of data; in the writing of the manuscript, and in the decision to publish the results.

\section{References}

1. Rakovan, J. Computer Programs for Drawing Crystal Shapes and Atomic Structures. Rocks Miner. 2018, 93, 60-64, doi:10.1080/00357529.2018.1383832

2. Weber, S. KrystalShaper-Crystal Morphology Editor/Viewer. JCrystalSoft. Available online: http://www.jcrystal.com/products/krystalshaper/index.htm (accessed on 12 June 2020).

3. Kraus, W.; Nolze, G. PowderCell 2.3-Powder Pattern Calculation from Single Crystal Data and Refinement of Experimental Curves. Available online: http://www.ccp14.ac.uk/ccp/web-mirrors/powdcell/a_v/v_1/powder/e_cell.html (accessed on 12 June 2020).

4. Momma, K.; Izumi, F. VESTA 3 for three-dimensional visualization of crystal, volumetric and morphology data. J. Appl. Crystallogr. 2011, 44, 1272-1276, doi:10.1107/S0021889811038970. Available online: https://jp-minerals.org/vesta/en/ (accessed on 12 June 2020).

5. Kaminsky, W. WinXMorph: A computer program to draw crystal morphology, growth sectors and cross sections with export files in VRML V2.0 utf8-virtual reality format. J. Appl. Crystallogr. 2005, 38, 566-567, doi:10.1107/S0021889805012148. Available online: http://cad4.cpac.washington.edu/WinXMorphHome/WinXMorph.htm (accessed on 12 June 2020).

6. Kaminsky, W. From CIF to virtual morphology using the WinXMorph program. J. Appl. Crystallogr. 2007, 40, 382-385, doi:10.1107/S0021889807003986.

7. Downs, R.T.; Hall-Wallace, M. The American Mineralogist crystal structure database. Am. Mineral. 2003, 88, 247-250. Available online: http://rruff.geo.arizona.edu/AMS/amcsd.php (accessed on 12 June 2020).

8. Available online: http://rruff.geo.arizona.edu/AMS/download.php?id=00802.amc\&down=amc (accessed on 12 June 2020).

9. Available online: http://rruff.geo.arizona.edu/AMS/AMC_text_files/18664_amc.txt (accessed on 12 June 2020). 